This is the final peer-reviewed accepted manuscript of:

Adverse events with sacubitril/valsartan in the real world: emerging signals to target preventive strategies from the FDA adverse event reporting system.

Milo Gatti; Ippazio Cosimo Antonazzo; Igor Diemberger; Fabrizio De Ponti; Emanuel Raschi.

European Journal of Preventive Cardiology, 2020 Apr 20.

The final published version is available online at:

http://dx.doi.org/10.1177/2047487320915663

Rights / License:

The terms and conditions for the reuse of this version of the manuscript are specified in the publishing policy. For all terms of use and more information see the publisher's website.

This item was downloaded from IRIS Università di Bologna (https://cris.unibo.it/)

When citing, please refer to the published version. 


\section{Adverse events with sacubitril/valsartan in the real world: emerging signals to target preventive strategies from the FDA Adverse Event Reporting System}

Milo Gatti ${ }^{1}$, Ippazio Cosimo Antonazzo ${ }^{1}$, Igor Diemberger*2, Fabrizio De Ponti ${ }^{1}$, Emanuel Raschi*1

${ }^{1}$ Department of Medical and Surgical Sciences, Alma Mater Studiorum, University of Bologna, Bologna, Italy

${ }^{2}$ Department of Experimental, Diagnostic and Specialty Medicine, University of Bologna, S.Orsola University Hospital, Bologna, Italy.

\section{Corresponding authors*}

Clinical enquiries:

Igor Diemberger, MD, $\mathrm{PhD}$

Institute of Cardiology, Department of Experimental, Diagnostic and Specialty Medicine, University of Bologna, Policlinico S.Orsola-Malpighi, Bologna, Italy.

Via Massarenti 9, 40138 Bologna, Italy

Tel.: +39-051-2143434

Fax: +39-051-6364216

E-mail: igor.diemberger@unibo.it

Pharmacological enquiries:

Emanuel Raschi, MD PhD

Pharmacology Unit, Department of Medical and Surgical Sciences, Alma Mater Studiorum, University of Bologna, Bologna, Italy

Via Irnerio 48, 40126, Bologna, Italy

Tel.: +390512091794

Fax: +390512091780

E-mail: emanuel.raschi@unibo.it 


\begin{abstract}
Aims: To characterize clinical priority of adverse events (AEs) with sacubitril/valsartan for targeting preventive measures.

Methods: We used the FDA Adverse Event Reporting System (worldwide pharmacovigilance database) to compare AEs recording sacubitril/valsartan as suspect with other cardiovascular drugs. Disproportionality analyses were performed by calculating the reporting odds ratios (RORs), deemed significant when the lower limit of the $95 \%$ confidence interval (LL95\%CI) $>1$. Clinical priority was assigned to AEs with significant disproportionality by scoring (range 0-10 points) five features (number of events, magnitude of LL95\%CI, mortality rate, important/designated medical event, biological plausibility).

Results: Sacubitril/valsartan was recorded in 20,021 reports, with 178 AEs associated with significant disproportionality: $71.9 \%, 25.9 \%$ and $2.2 \%$ were classified as weak, moderate and strong clinical priorities, respectively. Increased reporting emerged for several cardiovascular AEs, including "renal failure" $(\mathrm{N}=388$; LL95\%CI 2.26), "hyperkalaemia" (314; 2.42), and "angioedema" (309; 1.56). Sudden cardiac death (SCD) (priority score $=9$ points) was the only designated medical event with strong clinical priority. Notably, SCD occurred early after sacubitril/valsartan administration (average onset 124 days), with concomitant drugs known for pro-arrhythmic potential (e.g., amiodarone, escitalopram, mirtazapine, loop diuretics) in 26.2\% of records.

Conclusion: The increased cardiovascular reporting of sacubitril/valsartan in the real world was largely predictable from pre-approval evidence, underlying disease and likely patients' comorbidities. The unexpected reporting of SCD occurred well before the complete development of positive electrical remodelling induced by sacubitril/valsartan, and calls for stringent clinical monitoring (to reduce the proarrhythmic burden related to co-medications), and further investigation on appropriate combination with other preventive measures.
\end{abstract}

Keywords: sacubitril/valsartan; pharmacovigilance; disproportionality; sudden cardiac death. 


\section{Introduction}

The combination of sacubitril, the first-in-class angiotensin receptor-neprilysin inhibitor (ARNI), and valsartan, a well-known angiotensin receptor antagonist [1], was approved by the Food and Drug Administration (FDA) in 2015 and the European Medicines Agency (EMA) in 2016 for the management of patients affected by heart failure with reduced ejection fraction, based on the pivotal phase III PARADIGMHF trial [2], showing 20\% relative risk reduction in the primary composite endpoint (cardiovascular death or hospitalization for heart failure), as compared to enalapril alone. Notably, the divergence of incidence in primary outcome became evident only at day 180, and even later for individual outcomes.

This new combination coupled the blockade of detrimental effects associated with upregulation of reninangiotensin-aldosterone system promoted by valsartan, with beneficial effects on natriuretic peptide system due to neprilysin inhibition induced by sacubitril, overall resulting in increased natriuresis and diuresis, vasodilation, anti-proliferative effect and reduced sympathetic activity [1].

Overall, tolerability was predictable: symptomatic hypotension occurred more frequently in patients receiving the drug combination, whereas renal impairment (elevated creatinine $\geq 2.5 \mathrm{mg} / \mathrm{dL}$ ), hyperkalemia and cough (despite being common side effects) were significantly recorded in the enalapril group [2]. Furthermore, concerns emerged regarding the theoretical possibility of an increased risk for cognition, memory, or dementia-related adverse events (AEs), given that neprilysin inhibition may affect the brain clearance of amyloid-beta peptide [3-4].

Considering the recognized limitations and the observed methodological bias of a single clinical trial in fully evaluating the safety profile of drugs, especially in the detection of rare, unexpected and delayed AEs, spontaneous reporting systems (SRSs) represent a valuable source for post-marketing surveillance, allowing early identification of possible safety signals. Additionally, analysis of SRSs provides a broader perspective by collecting unpublished reports of AEs occurring in the real world, including multifaceted scenario of patients with comorbidities and poly-pharmacotherapy [5-6].

Safety assessment of sacubitril/valsartan appears a neglected clinical issue and, to the best of our knowledge, only a few case reports/series have preliminary described specific toxicities, mainly limited to muscular-, renal- and cognition-related AEs [3, 7-11].

We characterized the safety profile of sacubitril/valsartan, by analysing spontaneous reports submitted to the US FDA Adverse Event Reporting System (FAERS) database. The extracted AEs, potentially emerging as safety signals, were ranked in term of clinical priority level in order to identify and prioritize potential preventive measures. 


\section{Methods}

\section{Study design and data source}

The study was conceived as an observational, retrospective disproportionality analysis, a validated concept in pharmacovigilance to assess whether an association is likely to exist between sacubitril/valsartan and a given AE based on a differential reporting [12].

All reports recorded in FAERS between the second quarter (Q2) of 2015 (considering the US marketing approval of sacubitril/valsartan) and 2018Q2, in which the drug was considered the primary or secondary suspect, and reaching a pre-defined quality level, were included in our analysis. Other cardiovascular drugs were considered as control.

Disproportionality analysis was performed by calculating the reporting odds ratio (ROR) with relevant $95 \%$ confidence interval (CI), and considered statistically significant when the lower limit of the $95 \% \mathrm{CI}$ of the ROR exceeds 1. Disproportionality was calculated according to the traditional signalling criterion (when at least three cases of interest were reported) [12-14], and performed at the system organ class (SOC) and preferred term (PT) level to describe the spectrum of toxicities. Details concerning study design and disproportionality analysis are shown in Supplementary material -1 .

\section{Classification and prioritization of relevant disproportionality signals}

AEs emerging with significant disproportionality were ranked in terms of clinical priority as follows.

First, important medical events (IMEs) and designated medical events (DMEs) were selected among disproportionality signals using public lists developed and updated by the EMA [15-16]. IMEs are any AE showing seriousness features (death, life-threatening, hospitalization, disability or congenital anomaly). DMEs are rare, serious AEs associated with a high drug-attributable risk, which may constitute a safety issue under certain circumstances (e.g., high quality reports).

Second, IMEs and DMEs were prioritized according to three levels of clinical importance, by creating a semi-quantitative score assessing five different features: number of events reported, magnitude of the lower limit of the $95 \%$ CI of ROR, mortality rate, characterization as IMEs or DMEs, biological plausibility (Table 1). A score between 0-4, 5-7 or 8-10 identified respectively AEs with weak (green light), moderate (yellow light) or strong (red light) clinical priority.

No matter clinical relevance/priority, these findings were classified into four broad categories, according to the predictability of AEs:

Expected AEs: predictable events on the basis of sacubitril/valsartan mechanism of action, or anticipated from pre-marketing pivotal trials; 
Disease-related AEs: events for which underlying heart failure represents per se a risk factor (i.e. heart failure may increase the risk of supraventricular or ventricular arrhythmia);

Comorbidities-related AEs: events for which concomitant diseases or co-administered drugs represent risk factors (i.e. patients with heart failure are commonly affected by diabetes, or concomitant therapy with loop diuretics may lead to deafness or electrolyte abnormalities);

Unexpected AEs: unpredictable and previously unknown events. 


\section{Results}

Descriptive analysis

During the 3-year study period, a total of 16,331,098 FAERS reports were initially processed for drug codification and duplicate removal: 9,354,225 reports were finally retained, of which 1,149,398 included at least one cardiovascular drug; 20,021 reports (1.74\%; cases) mentioned sacubitril/valsartan as suspect agent, while 1,128,887 were non-cases (Supplementary Figure 1).

Demographic data are reported in Table 2. The reported country was US in $40.9 \%$ of records. Subjects aged $>60$ years old were the most represented, with male preponderance $(60.5 \%)$. Hospitalization and death occurred respectively in $14.4 \%$ and $12.1 \%$. Consumers represented the main source of reports in at least half of cases $(52.8 \%)$, followed by clinicians $(28 \%)$ and other health care professionals $(17.8 \%)$. The majority of reports was submitted during the 2017Q3-2018Q2 period (55.6\%).

\section{Disproportionality analysis}

The disproportionality analysis identified five areas of toxicity with statistically significant ROR: cardiac disorders $(\mathrm{N}=2559$; adjROR $=1.33 ; 95 \% \mathrm{CI} 1.27-1.39)$, ear and labyrinth disorders $(328 ; 1.15 ; 1.02-1.28)$, investigations $(3655 ; 1.33 ; 1.29-1.39)$, renal and urinary disorders $(1569 ; 1.40 ; 1.33-1.48)$ and vascular disorders $(3368 ; 2.29 ; 2.20-2.38)$, with consistent results for both crude and adjusted disproportionality (Supplementary Table 1, Supplementary Figure 2).

Overall, sacubitril/valsartan was recorded as suspect agent in $2288 \mathrm{PTs}$, of which $197(8.6 \%)$ showed a statistically significant ROR, representing $55.7 \%$ of cases. After accounting for indication bias and removal of medication errors, 178 PTs with significant disproportionality were finally retained. Sacubitril/valsartan was associated with higher reporting of renal failure $(\mathrm{N}=388 ; \operatorname{adjROR}=2.52 ; 95 \%$ CI 2.26-2.8), hyperkalaemia $(314 ; 2.74 ; 2.42-3.08)$, and angioedema $(309 ; 1.76 ; 1.56-1.98)$. Disease-related, expected and comorbidities-related AEs amounting respectively for $41.6 \%, 28.7 \%$ and $28.1 \%$ of PTs. Only three PTs (1.6\%) were classified as unexpected AEs, just including 31 cases $(0.1 \%)$ (Figure 1$)$.

\section{Clinical prioritization of relevant clinical signals}

Overall, 44 out of 178 PTs (24.7\%) showing statistically significant disproportionality were classified as IMEs (Table 3; Figure 1). Only four significant PTs (2.2\%) were identified as DMEs (Table 4; Figure 1), and none of these represented unexpected AEs. Collectively, 33 out of 62 DMEs (53.2\%) were recorded at least once with sacubitril/valsartan (Supplementary Table 2), with renal failure $(\mathrm{N}=388)$, acute kidney injury $(\mathrm{N}$ $=345)$ and angioedema $(\mathrm{N}=309)$ being the most common.

Based on clinical priority score, 128 (71.9\%), 46 (25.9\%) and 4 (2.2\%) PTs were classified as weak, moderate and strong clinical priority, respectively (Tables 3 and 4, Supplementary Table 3). Sudden cardiac 
death $(\mathrm{SCD})$ (points =9), sudden death (points =8), death (points =8) and ischaemic cardiomyopathy (points $=8$ ) emerged as strong clinical priorities.

Unexpected AEs showing statistically significant RORs (but not fulfilling criteria for strong prioritization) were: nipple pain $(\mathrm{N}=16$; adjROR $=5.59 ; 95 \%$ CI 3.01-9.84), hepatic cyst $(11 ; 2.27 ; 1.14-4.08)$ and pyoderma gangrenosum $(4 ; 22.23 ; 5.86-65.48)$. 


\section{Discussion}

To the best of our knowledge, this is the first real world study describing the worldwide reporting of AEs with sacubitril/valsartan, and one of its key findings is a major over-reporting of cardiovascular AEs. Previous studies were limited to local/national experiences [7-8] or focused on specific AEs such as dementia [3], thus providing only a narrow view of potential safety concerns emerging from post-marketing use of sacubitril/valsartan.

Disproportionality approaches are exponentially increasing in the recent literature, especially to investigate cardiovascular safety [17]. In our study, we attempted to move forward the application of statistical techniques by proposing a priority score based on well-defined criteria. Our score was developed to assign clinical relevance on signals and avoid unnecessary alarm. It may also be useful to assist clinicians to define toxicities emerging in daily practice that warrant further investigation to target preventive strategies, and support pharmacovigilance experts and regulatory agencies in routine signal detection activity to prioritize safety signals.

Overall, sacubitril/valsartan showed a predictable safety profile based on the known mechanism of action [1], anticipated common AEs found in PARADIGM-HF trial [2, 4], expected clinical complications, comorbidities and co-medications likely overlapping in patients with chronic cardiac insufficiency [18]. We interpreted the vast majority of cardiovascular AEs with significant disproportionality as strictly associated with heart failure per se (disease-related AEs) or with comorbidities and co-administered medications (comorbidities-related AEs), rather than with inherent toxicity of sacubitril/valsartan. In fact, considering therapeutic indication and the European Society of Cardiology (ESC) guidelines' recommendations, it is likely that patients eligible to sacubitril/valsartan are affected by advanced stages of cardiac insufficiency, thus being at higher risk of major cardiovascular events [18], a phenomenon known as "channeling" to be verified through pharmaco-epidemiological research.

Our score identified four AEs with strong clinical priority. These AEs, through expected and most likely related to the underlying heart failure, should not be overlooked considering the limited uptake of the drug in clinical practice coupled with the observed increasing reporting over time, as well as the observed discrepancy between patients enrolled in PARADIGM-HF trial and real-world use in the post-marketing registry [19-20].

Only apparently the finding of SCD is paradoxical, and an underlying drug-induced torsadogenic component cannot be excluded [21]. While there are no data on direct QT-prolonging potential sacubitril/valsartan at crediblemeds.org, the medication is likely to possess anti-arrhythmic properties considering the potential impact on reverse remodelling. In PARADIGM-HF trial, $20 \%$ reduction in cardiovascular death was attributable to SCD and death due to progressive heart failure, but became apparent only after day 180 and even later $[2,22]$. These findings have been reinforced by the results of a single centre study on implantable cardioverter defibrillator (ICD) carriers showing a reduction on anti-tachycardia interventions after 9 months 
of therapy with sacubitril/valsartan [23]. Furthermore, a positive electrical remodelling after switching from ACE-inhibitors to ARNI were reported, as documented by a reduction in QTc, T peak-T end interval and other measures of dispersion of ventricular repolarization [24].

Therefore, three main determinants may explain the possible occurrence of SCD with the use of sacubitril/valsartan: 1. late-onset of left ventricular reverse remodelling induced by ARNI; 2. concomitant ischaemic cardiomyopathy; 3. ethnicity. Different reports showed a clear temporal association between initiation of ARNI and early occurrence of ventricular arrhythmias [25-26]. Furthermore, an increased incidence of ventricular arrhythmias was recently reported in the first 12 months after initiation of sacubitril/valsartan, particularly in patients with ischaemic cardiomyopathy $(11 \%$ versus $37 \%, p=0.04)$ [27]. Notably, most of these events were recorded in the first 180 days after the shift in drug therapy. It is possible that neuro-hormonal and/or haemodynamic modification might be proarrhythmic in the acute phase, while after the occurrence of left ventricular reverse remodelling the outcome might improve. Evidence of a parallel behaviour between occurrence of reverse remodelling and reduction in the burden of ventricular arrhythmias may support this hypothesis [28].

In the 42 cases of SCD recorded in FAERS, the event occurred after a mean of 124 days, when left ventricular reverse remodelling induced by sacubitril/valsartan is still incompletely developed. We also recorded in a considerable proportion of cases different patient- and drug-related risk factors increasing the risk of torsade de pointes (TdP): use of antiplatelet/ anticoagulant agents, proxies of potential underlying ischaemic cardiomyopathy and atrial fibrillation, were reported in $42.9 \%$ of SCDs, while in $26.2 \%$ of cases, concomitant drugs known to increase directly (amiodarone, mirtazapine, escitalopram) or indirectly (loop diuretics via hypokalaemia) the pro-arrhythmic risk were recorded. Notably, cardiomyopathy/heart failure/acute myocardial ischemia represent per se risk factors for TdP.

In PARADIGM-HF trial [2], a higher prevalence of sudden death was reported in the Asia-Pacific region compared to remaining population (51.3\% vs. $33.0 \%$; $\mathrm{p}<0.00001)$, representing $25.5 \%$ of the overall SCDs recorded during the study period. Our data are aligned to this trend: cases of SCD, largely reported in men, accounted for $1.3 \%$ of overall records from Asia-Pacific region as compared to $0.3 \%$ in remaining population (6/453 vs. $36 / 10.561 ; p<0.001)$. Consequently, an underlying genetic susceptibility to ventricular arrhythmias strictly related to ethnicity cannot be excluded. Notably, the recently published PARAGON-HF trial [29] assessed the efficacy of sacubitril/valsartan in patients with heart failure and preserved ejection fraction, a scenario characterized by challenging management [30]: although in the overall population the study drug failed to reach a significant improvement, a better outcome was found in pre-specified subgroup analyses on Caucasian, female, and patients from Western Europe, thus reinforcing our hypothesis [31].

Although the prevalence of CRT (cardiac resynchronisation therapy) and ICD devices in PARADIGM-HF trial [2] was limited (respectively $6.8 \%$ and $14.8 \%$ ) compared to the relatively high prevalence of severe left ventricular dysfunction (two thirds of the patients presented a left-ventricular ejection fraction $<33 \%$ ), 
several authors are claiming the use of sacubitril/valsartan before or even in spite of ICD [32-33], given the significant reduction in cardiovascular and sudden death. In the wake of our data, there is an urgent need for high-quality data before replacing ICD implementation with sacubitril/valsartan administration: patients at high risk of SCD should be identified, and careful benefit-risk and cost-effectiveness assessment should be implemented [34]. In this regard, the results from the Improvement of Left Ventricular Ejection Fraction in ICD Patients Undergoing Therapy With Sacubitril/Valsartan (SAVE-ICD) prospective registry (NCT03935087) are strongly awaited.

The higher reporting of renal, ear and cognitive disorders (though partially expected) should not be overlooked. The considerable vasodilator effects exhibited by sacubitril/valsartan might impair renal perfusion, leading to clinically important rise in the serum creatinine level and acute renal injury. Clinicians should close monitor sacubitril/valsartan treated patients for the occurrence of acute kidney injury, considering that it represents a life-threatening DME. Moreover, chronic kidney disease (CKD) is commonly associated with heart failure, given that patients with CKD have greater prevalence of traditional heart failure risk factors as well as unique kidney-specific risk factors [35-37]. The significant disproportion for ear disorders may be likely associated with well-known AEs of loop diuretics (namely hypoacusis) used in the management of congestive heart failure [18].

Our analysis found that unexpected AEs only accounted for less than 2\% of PTs showing statistically significant disproportionality. Hepatic cyst and nipple pain exhibit weak clinical relevance and may be likely considered incidental findings in patients treated with sacubitril/valsartan. On the other hand, clinicians should be aware that pyoderma gangrenosum, although rare (only four cases recorded), might be a nonnegligible $\mathrm{AE}$ associated with sacubitril/valsartan. Pyoderma gangrenosum is an autoinflammatory neutrophilic dermatosis, which may progress to disabling complications, and was associated with certain medications (mainly colony-stimulating factors and small-molecule tyrosine kinase inhibitors) [38]. However, in our cases, concomitant drugs known to be associated with pyoderma gangrenosum were not found.

We acknowledge the limitations of our study, mainly related to the inherent nature of FAERS data, which do not allow to establish a causal relationship between drug exposure and occurrence of AE [12]. Given the lack of a denominator and the under-reporting phenomenon, the ROR and its magnitude cannot quantify the real risk in clinical practice, informing only an increased risk of $\mathrm{AE}$ reporting and not of $\mathrm{AE}$ occurrence. Consequently, incidence cannot be provided by spontaneous reports. Moreover, verification of events through clinical features, including laboratory and instrumental tests, comorbidities and adjustment of therapeutic regimens is limited.

However, FAERS is representative of the worldwide real-life use of drugs, which cannot be fully captured by clinical trials. Our attempt to control for main confounders (adjustment for co-medications and dataset restriction to cardiovascular area) and major bias (notoriety bias is not expected considering that no 
regulatory warnings were issued) strengthened the clinical implications of these findings. Finally, we applied an original score to assess the clinical impact of relevant disproportionality signals. Although its accuracy cannot be determined (and was not the aim of the work), our score is based on well-established criteria showing unquestionable clinical relevance (i.e. mortality rate, classification as DME or IME), including biological plausibility in order to prioritize AEs of clinical interest, thus also supporting identification of preventive strategies.

In conclusion, this real life safety assessment of sacubitril/valsartan found increased reporting of cardiovascular and renal AEs, although largely predictable and expected. Early occurrence of SCD is likely to reflect the underlying arrhythmogenic substrate and calls for careful monitoring, including therapy reconciliation strategies to minimize the pro-arrhythmic burden related to co-medications, particularly in the first weeks after the initiation of sacubitril/valsartan awaiting for delayed anti-arrhythmic effect of reverse remodelling.

Further investigations are warranted on appropriate preventive strategies: until differently demonstrated, initiation of sacubitril/valsartan should not preclude the use of implantable or external defibrillators to obtain the proper level of reverse remodelling, especially in high-risk patients.

In the meantime, considering the evolving use of sacubitril/valsartan, pharmacovigilance plays a key role in promoting risk-benefit assessment through active clinical surveillance, especially for unexpected AEs.

\section{Funding}

The author(s) disclosed receipt of the following financial support for the research, authorship, and/or publication of this article: D, ER and FDP are supported by institutional funds from Ricerca Fondamentale Orientata.

\section{Declaration of conflicting interests}

ID reports minor speaker fees from Bayer and Boehringer Ingelheim. ER reports personal fees from Novartis, outside the submitted work. MG, ICA, FDP have nothing to disclose.

Author contributions

MG contributed to the design of the work, analysis, interpretation of data, and drafted the manuscript. ICA contributed to the design, acquisition, analysis and interpretation of data, and critically revised the manuscript. ID and FDP contributed to the conception of the work, interpretation of data, and critically revised the manuscript. ER contributed to the conception and design of the work, analysis and interpretation 
of data, and critically revised the manuscript. All gave final approval and agree to be accountable for all aspects of work ensuring integrity and accuracy. 


\section{References}

1. D'Elia E, Iacovoni A, Vaduganathan M, et al. Neprilysin inhibition in heart failure: mechanisms and substrates beyond modulating natriuretic peptides. Eur J Heart Fail. 2017 Jun;19(6):710-717.

2. McMurray JJ, Packer M, Desai AS, et al; PARADIGM-HF Investigators and Committees. Angiotensin-neprilysin inhibition versus enalapril in heart failure. N Engl J Med. 2014 Sep 11;371(11):9931004.

3. Perlman A, Hirsh Raccah B, Matok I et al. Cognition- and Dementia-Related Adverse Effects With Sacubitril-Valsartan: Analysis of the FDA Adverse Event Report System Database. J Card Fail. 2018 Aug;24(8):533-536.

4. Cannon JA, Shen L, Jhund PS, et al; PARADIGM-HF Investigators and Committees. Dementiarelated adverse events in PARADIGM-HF and other trials in heart failure with reduced ejection fraction. Eur J Heart Fail. 2017 Jan;19(1):129-137.

5. Raschi E, Poluzzi E, Koci A, et al. Dipeptidyl peptidase-4 inhibitors and heart failure: Analysis of spontaneous reports submitted to the FDA Adverse Event Reporting System. Nutr Metab Cardiovasc Dis. 2016 May;26(5):380-6.

6. Raschi E, Mazzarella A, Antonazzo IC, et al. Toxicities with Immune Checkpoint Inhibitors: Emerging Priorities From Disproportionality Analysis of the FDA Adverse Event Reporting System. Target Oncol. 2019 Apr;14(2):205-221.

7. Moulis F, Rousseau V, Chebane L, et al. Serious adverse drug reactions with sacubitril/valsartan Entresto®: a French pharmacovigilance survey. Eur J Clin Pharmacol. 2018 Jul;74(7):983-984.

8. Adie S, Bitar A, Hanigan S, et al. Real-World Prevalence of Adverse Events After Initiating Sacubitril/Valsartan Compared With Angiotensin-Converting Enzyme Inhibitors or Angiotensin Receptor Blockers in Systolic Heart Failure. J Card Fail. 2019 May;25(5):412-413.

9. Rawla P, Raj JP, George SM, et al. A Rare Adverse Event of Rhabdomyolysis Caused by Sacubitril/Valsartan. Diseases. 2019 May 14;7(2). pii: E38.

10. Faber ES, Gavini M, Ramirez R, et al. Rhabdomyolysis After Coadministration of Atorvastatin and Sacubitril/Valsartan (Entresto $\left.{ }^{\mathrm{TM}}\right)$ in a 63-Year-Old Woman. Drug Saf Case Rep. 2016 Dec;3(1):14.

11. Bejan-Angoulvant T, Genet T, Vrignaud L, et al. Three case reports of involuntary muscular movements as adverse reactions to sacubitril/valsartan. Br J Clin Pharmacol. 2018 May;84(5):1072-1074.

12. Poluzzi E, Raschi E, Piccinni $\mathrm{C}$ et al. Data mining techniques in pharmacovigilance: analysis of the publicly accessible FDA adverse event reporting system (AERS). In: Data mining applications in 
engineering and medicine. Karahoca A, Ed. Croatia, InTech; 2012. p. 267 - 301. Available from: https://www.intechopen.com/books/data-mining-applications-in-engineering-and-medicine/data-miningtechniques-in-pharmacovigilance-analysis-of-the-publicly-accessible-fda-adverse-event-re [accessed 12.11.2019]

13. Slattery J, Alvarez Y, Hidalgo A. Choosing thresholds for statistical signal detection with the proportional reporting ratio. Drug Saf. 2013 Aug;36(8):687-92.

14. Raschi E, Poluzzi E, Salvo F, et al. Pharmacovigilance of sodium-glucose co-transporter-2 inhibitors: What a clinician should know on disproportionality analysis of spontaneous reporting systems. Nutr Metab Cardiovasc Dis. 2018 Jun;28(6):533-542.

15. European Medicines Agency. Inclusion/exclusion criteria for the "Important Medical Events" list. https://www.ema.europa.eu/en/documents/other/eudravigilance-inclusion/exclusion-criteria-importantmedical-events-list en.pdf [accessed 12.11.2019].

16. European Medicines Agency. Designated Medical Event (DME) list. 2016. https://www.ema.europa.eu/documents/other/designated-medical-event-dme-list_en.xls. 12.11.2019].

17. Salem JE, Manouchehri A, Moey M, et al. Cardiovascular toxicities associated with immune checkpoint inhibitors: an observational, retrospective, pharmacovigilance study. Lancet Oncol. 2018 Dec;19(12):1579-1589.

18. Ponikowski P, Voors AA, Anker SD, et al; Authors/Task Force Members; Document Reviewers. 2016 ESC Guidelines for the diagnosis and treatment of acute and chronic heart failure: The Task Force for the diagnosis and treatment of acute and chronic heart failure of the European Society of Cardiology (ESC) Developed with the special contribution of the Heart Failure Association (HFA) of the ESC. Eur J Heart Fail. 2016 Aug;18(8):891-975.

19. Kapelios CJ, Lainscak M, Savarese G, et al; Heart Failure Long-Term Registry Investigators. Sacubitril/valsartan eligibility and outcomes in the ESC-EORP-HFA Heart Failure Long-Term Registry: bridging between European Medicines Agency/Food and Drug Administration label, the PARADIGM-HF trial, ESC guidelines, and real world. Eur J Heart Fail. 2019 Nov;21(11):1383-1397.

20. Veenis JF, Brunner-La Rocca HP, Linssen GC, et al; CHECK-HF investigators. Age differences in contemporary treatment of patients with chronic heart failure and reduced ejection fraction. Eur J Prev Cardiol. 2019 Sep;26(13):1399-1407.

21. Poluzzi E, Raschi E, Diemberger I, De Ponti F. Drug-Induced Arrhythmia: Bridging the Gap Between Pathophysiological Knowledge and Clinical Practice. Drug Saf. 2017;40(6):461-464. 
22. Desai AS, McMurray JJ, Packer M, et al. Effect of the angiotensin-receptor-neprilysin inhibitor LCZ696 compared with enalapril on mode of death in heart failure patients. Eur Heart J. 2015 Aug 7;36(30):1990-7.

23. de Diego C, González-Torres L, Núñez JM, et al. Effects of angiotensin-neprilysin inhibition compared to angiotensin inhibition on ventricular arrhythmias in reduced ejection fraction patients under continuous remote monitoring of implantable defibrillator devices. Heart Rhythm. 2018 Mar;15(3):395-402.

24. Okutucu S, Sabanoglu C, Yetis Sayin B, et al. Switching from ramipril to sacubitril/valsartan favorably alters electrocardiographic indices of ventricular repolarization in heart failure with reduced ejection fraction. Acta Cardiol. 2018 Dec 4:1-6.

25. Okutucu S, Oto A. Electrical Storm after Initiating Sacubitril/Valsartan: Arrhythmic Paradox. Cardiology. 2019;142(1):24-25.

26. Vicent L, Juárez M, Martín I, et al. Ventricular Arrhythmic Storm after Initiating Sacubitril/Valsartan. Cardiology. 2018;139(2):119-123.

27. El-Battrawy I, Pilsinger C, Liebe V, et al. Impact of Sacubitril/Valsartan on the Long-Term Incidence of Ventricular Arrhythmias in Chronic Heart Failure Patients. J Clin Med. 2019 Oct 2;8(10).

28. Martens P, Nuyens D, Rivero-Ayerza M, et al. Sacubitril/valsartan reduces ventricular arrhythmias in parallel with left ventricular reverse remodeling in heart failure with reduced ejection fraction. Clin Res Cardiol. 2019 Oct;108(10):1074-1082.

29. Solomon SD, McMurray JJV, Anand IS, et al; PARAGON-HF Investigators and Committees. Angiotensin-Neprilysin Inhibition in Heart Failure with Preserved Ejection Fraction. N Engl J Med. 2019 Oct 24;381(17):1609-1620.

30. Memon MM, Yamani N, Asmi N, et al. Renin-angiotensin-aldosterone system inhibition in heart failure with mid-ranged ejection fraction: A systematic review and meta-analysis. Eur J Prev Cardiol. 2019 Apr 9:2047487319841937. doi: 10.1177/2047487319841937. [Epub ahead of print]

31. McMurray JJV, Jackson AM, Lam CSP, et al. Effects of Sacubitril-Valsartan, versus Valsartan, in Women Compared to Men with Heart Failure and Preserved Ejection Fraction: Insights from PARAGONHF. Circulation. 2019 Nov 17. doi: 10.1161/CIRCULATIONAHA.119.044491. [Epub ahead of print]

32. Zacà V. Sacubitril/valsartan or an implantable cardioverter-defibrillator in heart failure with reduced ejection fraction patients: a cost-effectiveness analysis. J Cardiovasc Med (Hagerstown). 2018 Oct;19(10):597-605.

33. Ehrlich JR. Do we still need ICDs if we have ARNi? Heart Rhythm. 2018 Mar;15(3):403-404. 
34. Perera K, Ademi Z, Liew D, et al. Sacubitril-valsartan versus enalapril for acute decompensated heart failure: a cost-effectiveness analysis. Eur J Prev Cardiol. 2019 Oct 4:2047487319878953. doi: 10.1177/2047487319878953. [Epub ahead of print]

35. Tuegel C, Bansal N. Heart failure in patients with kidney disease. Heart. 2017 Dec;103(23):18481853.

36. Seferović PM, Polovina M, Milinković I. A step forward in resolving an old issue: treatment of heart failure with preserved ejection fraction and renal dysfunction? Eur J Prev Cardiol. 2018 Aug;25(12):12631267.

37. Tsujimoto T, Kajio H. Efficacy of renin-angiotensin system inhibitors for patients with heart failure with preserved ejection fraction and mild to moderate chronic kidney disease. Eur J Prev Cardiol. 2018 Aug;25(12):1268-1277.

38. Wu BC, Patel ED, Ortega-Loayza AG. Drug-induced pyoderma gangrenosum: a model to understand the pathogenesis of pyoderma gangrenosum. Br J Dermatol. 2017 Jul;177(1):72-83. 


\section{Figure legend}

Figure 1 - Flowchart of disproportionality analysis performed at preferred term (PT) level. Green light: weak clinical priority; Yellow light: moderate clinical priority; Red light: strong clinical priority. AEs: adverse events; PTs: preferred terms. 
Table 1 - Semi-quantitative score assessing clinical priority of adverse events showing statistically significant disproportionality. A score between $0-4,5-7$ or 8-10 identifies respectively relevant signals with weak (green light), moderate (yellow light) or strong (red light) clinical priority.

\begin{tabular}{|l|c|c|c|}
\hline Clinical priority features & 2 points & 1 point & 0 points \\
\hline No. of events & $>50$ & $10-50$ & $<10$ \\
\hline $\begin{array}{l}\text { Magnitude of the lower } \\
\text { limit of the 95\% CI of the } \\
\text { ROR }\end{array}$ & $>5$ & $2-5$ & $1-2$ \\
\hline Mortality rate & $>50 \%$ & $25-50 \%$ & $<25 \%$ \\
\hline $\begin{array}{l}\text { Important or designated } \\
\text { medical events }\end{array}$ & Designated medical event & Important medical event & None \\
\hline Biological plausibility & $\begin{array}{c}\text { Established on the basis of } \\
\text { mechanism of action of } \\
\text { sacubitril/valsartan or heart } \\
\text { failure-related }\end{array}$ & $\begin{array}{c}\text { Supposed on the basis of } \\
\text { preclinical evidence or } \\
\text { presumed comorbidities or } \\
\text { concomitant drugs }\end{array}$ & $\begin{array}{c}\text { No clear biological } \\
\text { plausibility can be } \\
\text { retrieved }\end{array}$ \\
\hline
\end{tabular}

ROR: reporting odds ratio; $\mathrm{CI}$ : confidence interval. 
Table 2 - Demographic data.

\begin{tabular}{|c|c|c|}
\hline & Cases & Non-cases \\
\hline Overall number of reports & 20.021 & 1.128 .887 \\
\hline \multicolumn{3}{|l|}{$\overline{\text { Sex }}$} \\
\hline $\mathrm{F}$ & $5.738(28.7 \%)$ & $612.911(54.3 \%)$ \\
\hline $\mathrm{M}$ & $12.103(60.5 \%)$ & $427.585(37.9 \%)$ \\
\hline Missing & $2.180(10.8 \%)$ & $88.391(7.8 \%)$ \\
\hline \multicolumn{3}{|l|}{ Age (years) } \\
\hline$<10$ & $2(0.0 \%)$ & $12.627(1.1 \%)$ \\
\hline $10-19$ & $4(0.0 \%)$ & $13.857(1.2 \%)$ \\
\hline $20-29$ & $28(0.1 \%)$ & $19.254(1.7 \%)$ \\
\hline $30-39$ & $103(0.5 \%)$ & $34.655(3.1 \%)$ \\
\hline $40-49$ & $277(1.4 \%)$ & $67.497(6.0 \%)$ \\
\hline $50-59$ & $760(3.7 \%)$ & $140.178(12.4 \%)$ \\
\hline $60-69$ & $1.513(7.6 \%)$ & $203.267(18.0 \%)$ \\
\hline $70-79$ & $1.674(8.4 \%)$ & $179.412(15.9 \%)$ \\
\hline$\geq 80$ & $1.232(6.2 \%)$ & $96.720(8.6 \%)$ \\
\hline Missing & $14.428(72.1 \%)$ & $361.420(32.0 \%)$ \\
\hline \multicolumn{3}{|l|}{ Reporter Country } \\
\hline North America & $8.194(40.9 \%)$ & $806.116(71.4 \%)$ \\
\hline South America & $228(1.1 \%)$ & $18.507(1.6 \%)$ \\
\hline Europe & $2.096(10.5 \%)$ & $195.309(17.4 \%)$ \\
\hline Asia & $275(1.4 \%)$ & $66.020(5.8 \%)$ \\
\hline Oceania & $178(0.9 \%)$ & $7.865(0.7 \%)$ \\
\hline Africa & $43(0.2 \%)$ & $2.416(0.2 \%)$ \\
\hline Missing & $9.007(45.0 \%)$ & $32.654(2.9 \%)$ \\
\hline \multicolumn{3}{|l|}{ Reporter Type } \\
\hline Consumer & $10.578(52.8 \%)$ & $507.281(44.9 \%)$ \\
\hline Lawyer & $0(0.0 \%)$ & $14.685(1.3 \%)$ \\
\hline Medical Doctor & $5.600(28.0 \%)$ & $272.891(24.2 \%)$ \\
\hline Pharmacist & $232(1.2 \%)$ & $77.466(6.9 \%)$ \\
\hline Other healthcare professionals & $3.572(17.8 \%)$ & $213.875(18.9 \%)$ \\
\hline Missing & $39(0.2 \%)$ & $42.689(3.8 \%)$ \\
\hline \multicolumn{3}{|l|}{ Role code } \\
\hline Primary Suspect & $19.869(99.2 \%)$ & NA \\
\hline Secondary Suspect & $152(0.8 \%)$ & NA \\
\hline \multicolumn{3}{|l|}{ N. of co-reported drugs* } \\
\hline$<5$ & $16.325(79.6 \%)$ & NA \\
\hline$\geq 5$ & $4.186(20.4 \%)$ & NA \\
\hline \multicolumn{3}{|l|}{ Outcome } \\
\hline Congenital Anomaly & $0(0.0 \%)$ & $1.324(0.1 \%)$ \\
\hline Death & $2.425(12.1 \%)$ & $90.882(8.1 \%)$ \\
\hline Disability & $122(0.6 \%)$ & $16.148(1.4 \%)$ \\
\hline Hospitalization & $2.878(14.4 \%)$ & $281.194(24.9 \%)$ \\
\hline Life-Threatening & $359(1.8 \%)$ & $34.697(3.1 \%)$ \\
\hline Other & $3.253(16.2 \%)$ & $244.253(21.6 \%)$ \\
\hline Missing & $10.984(54.9 \%)$ & $460.389(40.8 \%)$ \\
\hline
\end{tabular}

NA: not applicable due to inability to identify an index drug.

* excluding uncodified medications and substances not formally considered as medications. 
Table 3 - Designated medical events showing statistically significant disproportionality.

\begin{tabular}{|l|c|c|c|c|c|}
\hline $\begin{array}{c}\text { Designated medical } \\
\text { events }\end{array}$ & $\begin{array}{c}\text { No. } \\
\text { cases }\end{array}$ & ROR adj & Death & $\begin{array}{c}\text { Biological } \\
\text { plausibility }\end{array}$ & $\begin{array}{c}\text { Priority level } \\
\text { (score) }\end{array}$ \\
\hline RENAL FAILURE & 388 & $2,52(2,26-2,8)$ & 92 & Expected & Moderate (7) \\
\hline ANGIOEDEMA & 309 & $1,76(1,56-1,98)$ & 10 & Expected & Moderate (6) \\
\hline $\begin{array}{l}\text { VENTRICULAR } \\
\text { FIBRILLATION }\end{array}$ & 46 & $1,78(1,29-2,38)$ & 22 & Disease-related & Moderate (6) \\
\hline $\begin{array}{l}\text { SUDDEN CARDIAC } \\
\text { DEATH }\end{array}$ & 42 & $12,82(8,53-19,13)$ & 42 & Disease-related & Strong (9) \\
\hline
\end{tabular}

ROR adj: adjusted reporting odds ratio (see text for details). 